\title{
One-step Synthesis of Ultrasmall Platinum Nanoparticles Supported on Amino-Functionalized Graphene to Create an Electrochemical Molecularly Imprinted Polymer Sensor for Fluxapyroxad
}

\author{
Ling Shi ${ }^{1}$, Jie Duan ${ }^{1}$, Shufang $X u^{1}$, Hongping Yang ${ }^{2}$, Guangming Yang ${ }^{1, *}$ \\ ${ }^{1}$ Engineering Research Center for Processing and Quality Control of Local Characteristic Food and \\ Consumer Goods of High Education in Yunnan Province, College of Science, Honghe University, \\ Mengzi 661199, PR China \\ ${ }^{2}$ Library, Honghe University, Mengzi 661199, PR China \\ *E-mail: yangguangmingbs@126.com
}

doi: $10.20964 / 2021.07 .19$

Received: 22 February 2021 / Accepted: 15 April 2021 / Published: 31 May 2021

In this work, a novel electrochemical molecularly imprinted polymer (MIP) sensor for fluxapyroxad (FP) was constructed. This MIP sensor was based on indole-6-carboxylic acid (6-IAA) and platinum (Pt) nanoparticles (NPs) supported amino-functionalized graphene $\left(\mathrm{NH}_{2}-\mathrm{r}-\mathrm{GO}\right)$. Massive and ultrasmall $\mathrm{Pt}$ NPs (2-3 nm) were supported on $\mathrm{NH}_{2}-\mathrm{r}-\mathrm{GO}$, which was easily prepared using a simple, aqueous phase method. Pt NP was firstly generated using ascorbic acid as the reducant and hexadecylpyridinium chloride as the structure-directing agent; then, NPs were immediately loaded on the surface of the coexisting $\mathrm{NH}_{2}$-r-GO. Loading allowed for the interreaction between the amino group and Pt to form the hybrid nanocomposite. This hybrid material was then used to modify a glassy carbon electrode (GCE) surface, resulting in a Pt-NPs-NH 2 -r-GO / GCE. After this modification, FP was imprinted on the aforementioned electrode surface using cyclic voltammetry, with 6-IAA as the functional monomer. Finally, FP was eluted from the polymer, resulting in the MIP sensor. After rebinding the template molecules $\mathrm{Fe}(\mathrm{CN}) 6^{3-14-}$ was used to measure the impedance of the MIP sensor. Results indicated an excellent response for FP across a linear range of $1.0 \times 10^{-9}$ to $1.3 \times 10^{-5} \mathrm{~mol} \mathrm{~L}^{-1}$; the detection limit was determined to be $1.0 \times 10^{-10} \mathrm{~mol} \mathrm{~L}^{-1}(\mathrm{~S} / \mathrm{N}=3)$. This sensor was also used to detect FP in real samples, with results indicating it was a reliable sensor for FP.

Keywords: Electrochemical sensor; Fluxapyroxad; Molecularly imprinted polymer; Indole-6carboxylic acid; Pt nanoparticles; Amino group functionalized-graphene 
(C) 2021 The Authors. Published by ESG (www.electrochemsci.org). This article is an open access article distributed under the terms and conditions of the Creative Commons Attribution license (http://creativecommons.org/licenses/by/4.0/). 